\title{
Sudden intrauterine death associated with furcate insertion of the umbilical cord
}

\author{
Laberke, P J ; Deubler, G
}

\begin{abstract}
In the case described, a completely uncomplicated pregnancy ended with a fatal outcome. Intrauterine fetal death, which was diagnosed prepartum at 40 weeks of gestation, was caused by hemorrhage of the fetus into the amniotic fluid following rupture of the umbilical vein at the site of furcate insertion of the umbilical cord. This insertion anomaly accompanied by rupture of a vessel occurs only very rarely but represents a placental cause of an abrupt and unpredicted end of a pregnancy. Although this process involved trauma, from a medico-legal point of view, there was no sign of external impact and death could, therefore, be classified as natural.
\end{abstract}

DOI: https://doi.org/10.1007/s00414-008-0295-6

Posted at the Zurich Open Repository and Archive, University of Zurich ZORA URL: https://doi.org/10.5167/uzh-25357

Journal Article

Published Version

Originally published at:

Laberke, P J; Deubler, G (2009). Sudden intrauterine death associated with furcate insertion of the umbilical cord. International Journal of Legal Medicine, 123(6):509-512.

DOI: https://doi.org/10.1007/s00414-008-0295-6 


\title{
Sudden intrauterine death associated with furcate insertion of the umbilical cord
}

\author{
P. J. Laberke • G. Deubler
}

Received: 28 May 2008 / Accepted: 29 October 2008/Published online: 26 November 2008

(C) Springer-Verlag 2008

\begin{abstract}
In the case described, a completely uncomplicated pregnancy ended with a fatal outcome. Intrauterine fetal death, which was diagnosed prepartum at 40 weeks of gestation, was caused by hemorrhage of the fetus into the amniotic fluid following rupture of the umbilical vein at the site of furcate insertion of the umbilical cord. This insertion anomaly accompanied by rupture of a vessel occurs only very rarely but represents a placental cause of an abrupt and unpredicted end of a pregnancy. Although this process involved trauma, from a medico-legal point of view, there was no sign of external impact and death could, therefore, be classified as natural.
\end{abstract}

Keywords Stillbirth · Intrauterine fatal death · IUFD . Insertio furcata $\cdot$ Umbilical cord

\section{Introduction}

The investigation of sudden unexpected death during pregnancy, intrapartum, and postpartum is difficult. In all of these cases, a complete postmortem examination should be carried out to differentiate sudden and unexpected deaths due to natural causes and cases of unnatural death $[2,4,5,9]$.

P. J. Laberke $(\triangle)$

Institute of Legal Medicine, University of Zurich,

Winterthurerstr. 190/52,

CH-8057 Zurich, Switzerland

e-mail: patrick.laberke@irm.uzh.ch

G. Deubler

Institute of Pathology, Reutlingen District Hospitals,

Steinenbergstr. 31,

72764 Reutlingen, Germany
The cause of individual cases of intrauterine fetal death (IUFD) may be found to be natural, unnatural, or may remain unexplained. Amongst the unnatural causes of IUFD, blunt injury to the maternal abdomen resulting from road traffic accidents is the most common cause in the third trimester [11]. The possibility of illegal or incorrectly performed abortion as a cause also needs to be considered, especially in the first and second trimesters, but because, presumably, the true nature of considerable numbers of such cases is not identified, reliable statistics for this situation are not available [6, 12]. A wide variety of methods are used to achieve illegal abortion, for example, deliberate blunt trauma to the abdomen, manipulation with instruments, sometimes causing perforation, insufflation of the uterus with air or liquids, and occasionally, the intake of medicines, drugs, or other chemical substances.

The German Federal Statistical Office ("Statistisches Bundesamt") registers only those cases of IUFD due to natural or unknown causes in which the fetus weighs more than 500 g. About 3,000 stillbirths per year have been recorded over the past 10 years in Germany [12]. The large number of cases occurring during early pregnancy is not included because of the weight specification.

More than half (50.5-59.0\%) of the cases of IUFD due to natural causes occurred as a result of anoxia associated with a disturbance of placental function, i.e., acute or chronic placental insufficiency $[7,14,16]$. Infection $(2.1-$ $21.0 \%$ ) and congenital abnormalities and malformation (11.4-13.4\%) are also common causes. The cause of death remains unclear in $5.4-25.0 \%$ of cases of IUFD; in some cases, due to a lack of detailed investigation of the fetus and placenta.

This paper describes a rare lethal complication involving the umbilical cord and discusses the medico-legal implications of a traumatic event during pregnancy. 


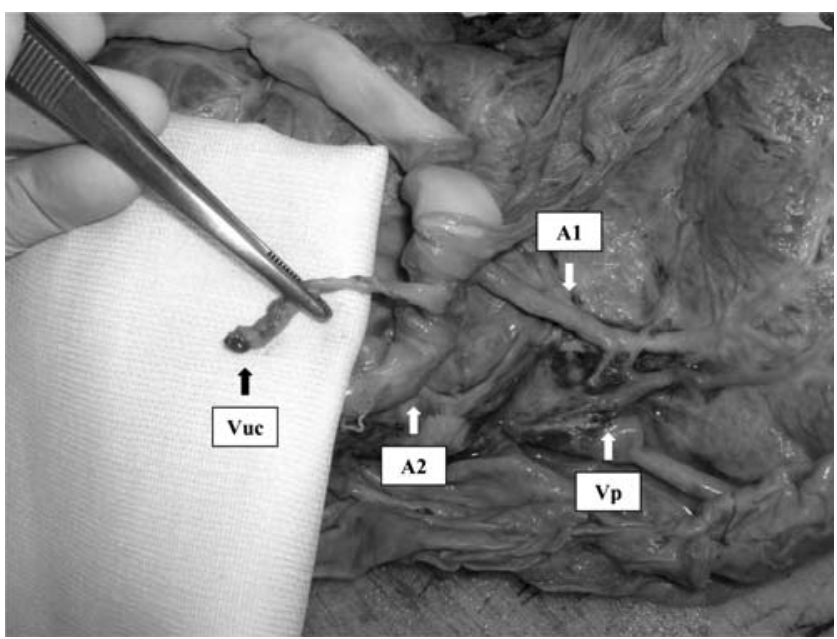

Fig. 1 Area of insertion of the umbilical cord into the placenta. AI umbilical artery, $A 2$ umbilical artery, Vuc umbilical vein (remnant attached to cord), $V p$ umbilical vein (remnant attached to placenta)

\section{Case history}

A 22-year-old woman, gravida 2, para 0 , attended the obstetrician's consulting rooms for a routine antenatal examination at 40 weeks +2 days gestation. The pregnancy had been uneventful and uncomplicated and there was no relevant history.

On CTG monitoring, it was found impossible to detect fetal heart sounds. The patient reported having last noticed fetal movements the previous day. There was no vaginal bleeding. The patient was referred immediately to the hospital where the diagnosis of IUFD was confirmed. The dead fetus was delivered vaginally. The liquor was heavily blood-stained and blood was seen on the placenta, membranes, and cord insertion.

\section{Autopsy findings}

Fetus

The fetus was a mature female infant weighing 3,220 g. Rigor mortis and postmortem lividity were mild, and early signs of maceration were seen. There were no out- ward signs of congenital malformation or trauma. The internal organs were pale but otherwise normal for gestational age.

Placenta and umbilical cord

The placenta weighed $580 \mathrm{~g}$. The attached umbilical cord contained three vessels along the whole of its length. The cord vessels were found to lie free in the amniotic cavity over a length of $4.5 \mathrm{~cm}$ above the site of insertion of the cord, which is the abnormality known as furcate insertion of the umbilical cord. One vessel had been torn away at the point of insertion (Fig. 1, Vuc), and a large amount of blood was seen in this area (Fig. 1, Vp).

Histology and immunohistochemistry

Histological investigations revealed the internal organs to be largely normal for gestational age, but blood-depleted. The bronchial system contained numerous erythrocytes and occasional skin cells derived from aspiration of the bloodstained amniotic fluid. There was no evidence of any internal condition that could have contributed to the cause of death.

The presence of three cord vessels was confirmed histologically. No inflammatory infiltrates were found. The three vessels ran singly in the area of the furcate insertion, covered by amniotic epithelium but not by Wharton's jelly. Extensive fresh blood clots were seen at the site of the rupture, but there was no increase in inflammatory cells.

The ruptured vessel was identified as a vein by connective tissue staining (elastica van Gieson stain) and immunohistological staining for smooth muscle (sm-actin, Dako, Hamburg). The placental parenchyma exhibited the histological structure of a mature placenta. No morphological evidence of acute or chronic placental insufficiency or amnionitis was observed.

\section{Cause of death}

The findings of autopsy and investigation of the placenta revealed the cause of death to be fetal hypovolemic shock.
Fig. 2 Variations in the mode of insertion of the umbilical cord (modified). a normal insertion, b aberrant vessel, c velamentous insertion, $\mathbf{d}$ furcate insertion
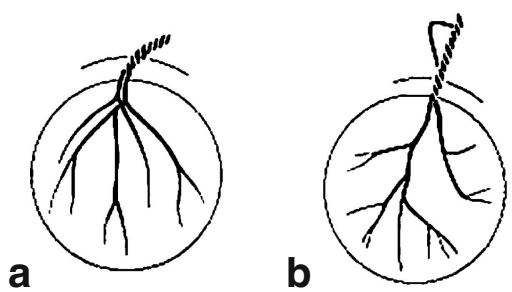

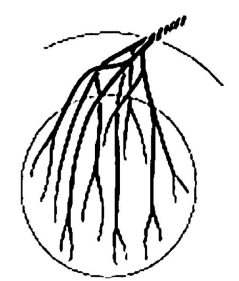

d

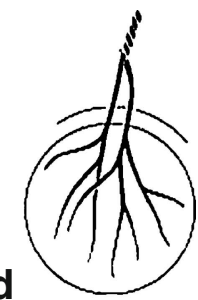


This had resulted from traumatic rupture of the umbilical vein associated with the rare anatomical abnormality known as furcate insertion of the umbilical cord. Examination of the mother and the results of the autopsy did not reveal any indications of any external influence.

\section{Variations in insertion of the umbilical cord}

Anomalies of cord insertion (Fig. 2) are seen in $30-50 \%$ of pregnancies with no known risk factors [14]. These are mainly variations of the normal condition in which the site of insertion is paracentral and complications are rare. If paracentral insertion, which is clinically irrelevant, is excluded, then only about $9 \%$ of all singleton pregnancies can be said to exhibit a pathological insertion of the cord [3].

In the case of marginal insertion of the cord (approximately 7\%), there is an increased risk of impairment of fetoplacental perfusion. However, the structure of the cord itself here is normal and the vessels are surrounded by Wharton's jelly. A markedly increased risk of the fetal circulation being compromised and vascular injury is associated with the rarer insertion anomalies such as velamentous insertion (1-2\%) and aberrant vessels $(2-3 \%)$ [1].

In velamentous insertion of the cord, the umbilical vessels run through the membranes, sometimes for several centimeters, branching here and being devoid of Wharton's jelly in this area. This anomaly poses the risk of compression of the vessels, leading to severe impairment of perfusion, which may be associated with thrombosis. In the case of placenta previa, the vessels may rupture during parturition [1].

When aberrant vessels are present, vascular loops lie free in the amniotic cavity. The complications are largely similar to those associated with furcate insertion of the cord.

Furcate insertion of the cord was first described by Hyrtl in 1870 [8]. It occurs with an incidence of $0.1 \%$, being one of the rarest pathological insertion anomalies. The cord separates into two or three vessels in the proximity of the placenta and these are not surrounded by Wharton's jelly, but rather by amniotic epithelium alone. The lack of Wharton's jelly, therefore, produces a situation similar to that in velamentous insertion or aberrant vessels that is accompanied by a risk of vascular rupture as a result of fetal movements. The insertion itself may be central, paracentral, marginal, or velamentous with combinations of these also being possible [10, 14].

Despite the risks described, furcate insertion is not always associated with complications, and most affected pregnancies progress uneventfully and end with the delivery of a healthy child [1].

\section{Discussion}

Lethal complications involving the umbilical cord are found in $7.7-11.4 \%$ of stillbirths and $0.06 \%$ of all pregnancies $[7,14,16]$.

Although some of the insertion anomalies described can be detected by ultrasound $[3,15]$, it would seem that it is not usually possible to diagnose furcate insertion prenatally. When fetal death occurs completely without warning at the end of a regularly monitored and uneventful pregnancy, the obstetrician is likely to be asked by the patient whether it would not have been possible to avoid this lethal outcome. It is, therefore, in the interest of both doctor and patient to perform an autopsy in all cases of IUFD [5, 13]. Blunt abdominal trauma, in particular, must always be excluded as an unnatural cause of death in late pregnancy. By contrast, legal and illegal abortion plays the most important role with regard to pregnancies that terminate during the first and second trimester [12].

In the case described, the rupture of the umbilical vein leading to fatal hemorrhage was provoked by fetal movements in the amniotic cavity and can accordingly be considered traumatic. This "internal" and accidental trauma is not of relevance with regard to feticide and can be classified as a natural death. An accusation of professional negligence on the part of the parents would not be justified, especially as all antenatal examinations had been performed state-of-the-art, these kinds of anomalies cannot reliably be detected as a matter of routine and there are no guidelines concerning preventative measures in such cases.

\section{References}

1. Baergen RN (2005) Manual of Benirschke and Kaufmann's pathology of the human placenta. Springer, Berlin, pp 263-274

2. Bajanowski T, Vennemann M, Bohnert M, Rauch E, Brinkmann B, Mitchell EA, GeSID Group (2005) Unnatural causes of sudden unexpected deaths initially thought to be sudden infant death syndrome. Int J Legal Med 119:213-216

3. Benirschke K, Kaufmann P, Beargen R (2006) Pathology of the human placenta. Springer, Berlin, pp 401-426

4. Bohnert M, Große Perdekamp M, Pollak S (2004) Three subsequent infanticides covered up as SIDS. Int J Legal Med 119:31-34

5. Dirnhofer R, Sigrist T (1983) Intra partum death or infanticide? A contribution on intrapartal asphyxia. Z Rechtsmed 91:145-151

6. Drasch G, Meyer Lv (1978) Dokumentation of autopsies from the Institute of Legal Medicine of Munich 1911-1974 and interpretation of the violent death causes. Z Rechtsmed 82:157-164

7. Golding J (1993) Epidemiology of fetal and neonatal death. In: Fetal and neonatal pathology, 2nd edn. Springer, London, pp 165181

8. Hyrtl J (1870) Die Blutgefäße der menschlichen Nachgeburt in normalen und abnormalen Verhältnissen. Braunmüller, Wien

9. Ohshima T, Kondo T, Lin Z, Nagano T (1993) Suspected maternal infanticide in a case of hydranencephaly. Int J Legal Med 105: 351-354 
10. Ottow B (1923) Über die Insertio furcata der Nabelschnur. Arch Gynecol Obstet 118:378-382

11. Schneider H (1993) Trauma in der Schwangerschaft. Arch Gynecol Obstet 253:4-14

12. Statistisches Bundesamt Deutschland (2006) Totgeborene insgesamt $1990-2005$

13. Steinbiss W (1928) Tod infolge einer Placenta cervicalis accreta unter falschem Verdacht auf kriminellen Abort. Z Rechtsmed $12: 234-240$
14. Vogel M (1997) Pathologie der Schwangerschaft, der Plazenta und des Neugeborenen. In: Remmele W (ed) Pathologie. 2nd edn. Springer, Heidelberg, pp 369-460

15. Weissman A, Jakobi P, Bronshtein M, Goldstein I (1994) Sonographic measurements of the umbilical cord and vessels during normal pregnancies. J Ultrasound Med 13:11-14

16. Wessel J, Gerhold W, Unger M, Lichtenegger W, Vogel M (1992) Nabelschnurkomplikationen als Ursache des intrauterinen Fruchttodes. Z Geburtshilfe Perinatol 196(4):173-176 